\title{
Demonstration of Adiabatic Variational Quantum Computing with a Superconducting Quantum Coprocessor
}

\author{
Ming-Cheng Chen, ${ }^{1,2}$ Ming Gong $\odot,{ }^{1,2}$ Xiaosi Xu $\odot,{ }^{3}$ Xiao Yuan, ${ }^{3}$ Jian-Wen Wang, ${ }^{1,2}$ Can Wang,,${ }^{1,2}$ \\ Chong Ying $\odot,{ }^{1,2}$ Jin Lin, ${ }^{1,2}$ Yu Xu, ${ }^{1,2}$ Yulin Wu, ${ }^{1,2}$ Shiyu Wang, ${ }^{1,2}$ Hui Deng, ${ }^{1,2}$ Futian Liang $\odot,{ }^{1,2}$ \\ Cheng-Zhi Peng, ${ }^{1,2}$ Simon C. Benjamin, ${ }^{3}$ Xiaobo Zhu, ${ }^{1,2}$ Chao-Yang Lu®, ${ }^{1,2}$ and Jian-Wei Pan ${ }^{1,2}$ \\ ${ }^{1}$ Shanghai Branch, National Laboratory for Physical Sciences at Microscale and Department of Modern Physics, \\ University of Science and Technology of China, Shanghai 201315, China \\ ${ }^{2}$ CAS Center for Excellence in Quantum Information and Quantum Physics, University of Science and Technology of China, \\ Hefei, Anhui 230026, China \\ ${ }^{3}$ Department of Materials, University of Oxford, Parks Road, Oxford OX1 3PH, United Kingdom
}

(Received 31 July 2019; accepted 22 September 2020; published 26 October 2020)

\begin{abstract}
Adiabatic quantum computing enables the preparation of many-body ground states. Realization poses major experimental challenges: Direct analog implementation requires complex Hamiltonian engineering, while the digitized version needs deep quantum gate circuits. To bypass these obstacles, we suggest an adiabatic variational hybrid algorithm, which employs short quantum circuits and provides a systematic quantum adiabatic optimization of the circuit parameters. The quantum adiabatic theorem promises not only the ground state but also that the excited eigenstates can be found. We report the first experimental demonstration that many-body eigenstates can be efficiently prepared by an adiabatic variational algorithm assisted with a multiqubit superconducting coprocessor. We track the real-time evolution of the ground and excited states of transverse-field Ising spins with a fidelity that can reach about $99 \%$.
\end{abstract}

DOI: 10.1103/PhysRevLett.125.180501

Solving quantum ground-state problems is key for applications in chemistry [1-4], materials science [5-7], and beyond [8-10]. It is believed that there are no classical algorithms for efficiently solving the general ground-state problems due to the notorious sign problem [11]. In contrast, quantum computing alleviates the sign problem by directly operating with complex-value quantum states and thus may provide a potential speed-up [12,13]. Adiabatic state preparation is a natural approach for quantum ground-state problems [1,9,14]. Starting from the ground state of a simple initial Hamiltonian $H_{0}$, such as $|+\rangle^{\oplus N}$ of $H_{0}=-\sum_{i=1}^{N} \sigma_{x}^{i}$, we would evolve to a complex target Hamiltonian $H_{T}$. The quantum adiabatic theorem [15] guarantees that if the change is sufficiently slow, the system will stay at its instantaneous eigenstate and ultimately reach the ground state of $H_{T}$. In certain cases it may be possible to realize the evolving Hamiltonian directly with suitable hardware; however, in many applications, including chemistry-related tasks, $H_{T}$ involves nonlocal connectivity and high-degree terms that are infeasible to implement.

We might resort instead to the flexibility of a fully digitized gate-based quantum circuit. In this context, the recent quantum-classical hybrid algorithms, such as quantum approximate optimization algorithm [16] and the variational quantum eigensolver [17], are a promising route toward useful exploitation of small- and medium-scale quantum computers. Generally, a hybrid algorithm would encode a "trial" quantum state via a shallow parameterized quantum circuit (i.e., the quantum coprocessor). A governing classical computer iteratively adjusts the parameters and monitors the output of the quantum circuit, ultimately seeking the parameters for which the output matches the ground state of $H_{T}$. The challenge is to achieve this in a fashion that can scale to the case of hundreds or thousands of parameters; the feasibility of this task is an active area of study [3].

Here, we marry together the adiabatic protocol with a circuit-based noisy intermediate-scale quantum (NISQ) coprocessor. This is enabled by recent theoretical work showing that the general dynamical evolution of (both closed and open) physical systems can be efficiently simulated with variational quantum algorithms $[18,19]$. We demonstrate this variational algorithm to the adiabatic dynamics of a 1D Ising spin chain model undergoing transverse-field driving.

In our algorithm, an $N$-qubit quantum state $|\psi\rangle$ is approximately parameterized by the output $|\phi\rangle$ of a lowdepth quantum gate circuit. This circuit ansatz consists of some fixed single-qubit and two-qubit gates and $L$ embedding variable single-qubit rotation gates of angles $\vec{\theta}=\left(\theta_{1}, \theta_{2}, \ldots, \theta_{L}\right)$, where the number of parameters $L \sim \operatorname{poly}(N)$. That is $|\psi(t)\rangle \approx|\phi(\vec{\theta})\rangle=U(\vec{\theta})|0\rangle$.

Then the dynamics of a quantum state can be mapped to the evolution of the parameters $\vec{\theta}$. The $2^{N}$-dimensional Schrödinger equation of quantum state $|\psi(t)\rangle$, 
(a)

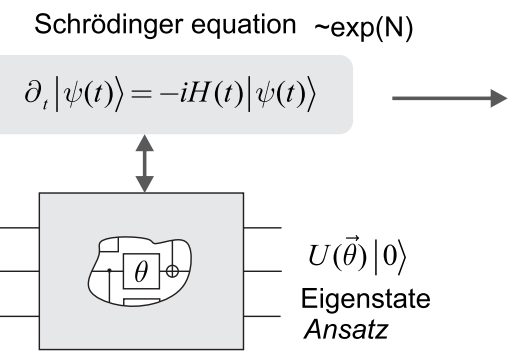

ODE $\sim \operatorname{poly}(\mathrm{N})$

(b)
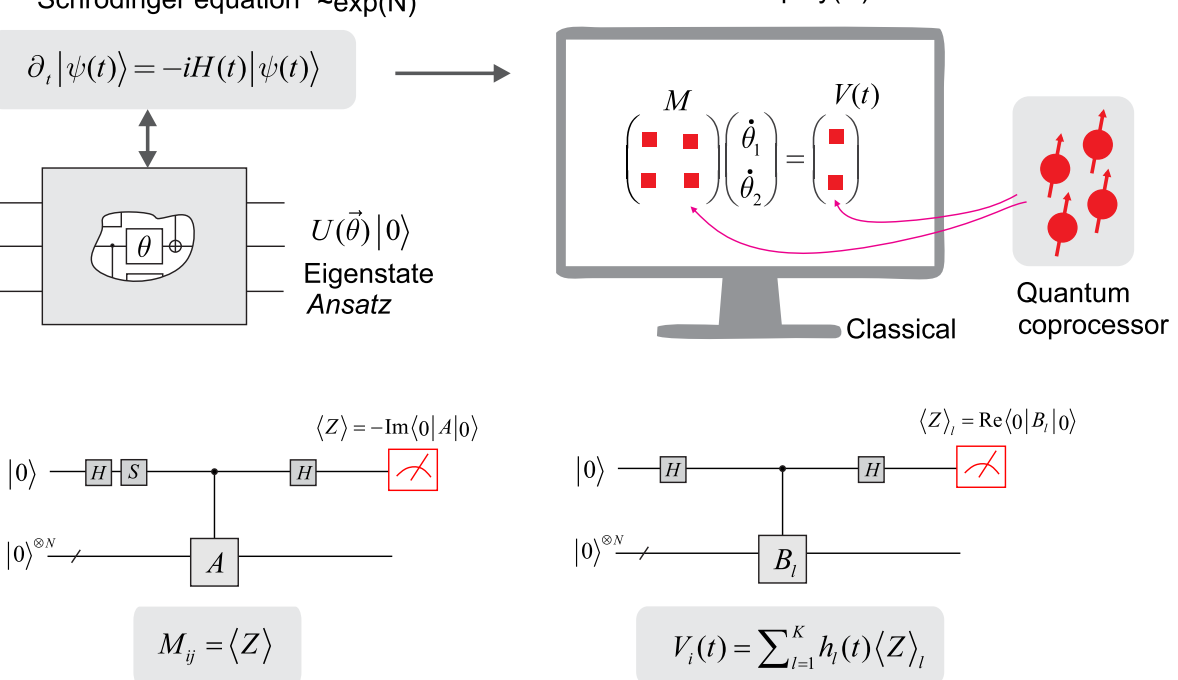

FIG. 1. Variational quantum simulation of dynamics. (a) Sketch of the variational algorithm. A shallow circuit ansatz $U(\vec{\theta})|0\rangle$ is used to approximate the true wave function $|\psi(t)\rangle$ with a polynomial number of parameters $\vec{\theta}(t)$. The Schrödinger equation is mapped to an ordinary differential equation (ODE) according to $M \cdot(\partial / \partial t) \vec{\theta}(t)=V(t)$. The ODE's coefficients $M$ and $V(t)$ are estimated on a quantum coprocessor and the ODE is numerically integrated on a classical computer. (b) The Hadamard test circuits are used to estimate $M_{i j}$ and $V_{i}(t)$ on the quantum coprocessor, respectively. In the circuit, $H$ is the Hadamard gate and $S$ is the phase gate. The unitary operator $A=\left(\partial_{\theta_{i}} U^{\dagger}\right)\left(\partial_{\theta_{j}} U\right)$ and $B_{l}=\left(\partial_{\theta_{i}} U^{\dagger}\right) P_{l} U$, where $U$ is the circuit of ansatz and $P_{l}$ is the Pauli term in the time-dependent Hamiltonian $H(t)=\sum_{l=1}^{K} h_{l}(t) P_{l}$.

$$
\frac{\partial}{\partial t}|\psi(t)\rangle=-i H|\psi(t)\rangle(\hbar=1)
$$

becomes an $L$-dimensional ordinary differential equation (ODE) of the variable $\vec{\theta}$,

$$
\frac{\partial}{\partial t} \vec{\theta}(t)=M^{-1} \cdot V(t)
$$

where the elements of matrix $M$ and vector $V$ are, respectively [18],

$$
\begin{aligned}
M_{i, j} & =-\operatorname{Im}\left(\frac{\partial\langle\phi(\vec{\theta})|}{\partial \theta_{i}} \frac{\partial|\phi(\vec{\theta})\rangle}{\partial \theta_{j}}\right), \\
V_{i}(t) & =\operatorname{Re}\left(\frac{\partial\langle\phi(\vec{\theta})|}{\partial \theta_{i}} H(t)|\phi(\vec{\theta})\rangle\right) .
\end{aligned}
$$

If we know the coefficients $M$ and $V$ in the ODE, the evolution of circuit parameters $\vec{\theta}(t)$ can be efficiently solved on a classical computer in polynomial time $\sim O\left(L^{2}\right)$ by direct numerical integration. However, the coefficients of ODE are related to inner products of quantum state $|\phi\rangle$, which are generally hard for a classical computer to estimate due to its $2^{N}$ dimension.

When noting that these coefficients can be efficiently evaluated by a quantum computer, the ODE will be solved efficiently through classical-quantum hybrid iterations. For each step at time $t$, a quantum computer is used to estimate the ODE's coefficients $M$ and $V$ and then a classical computer is used to integrate the equation with time interval $\delta t$ and update circuit parameters: $\vec{\theta}(t+\delta t)=$ $\vec{\theta}(t)+M^{-1} \cdot V(t) \times \delta t$.

Next, we describe the related quantum circuit to estimate the ODE's coefficients. The matrix elements $M_{i, j}$ are related to the imaginary of an inner product of the form $\langle 0|A| 0\rangle$, where $A=\left(\partial_{\theta_{i}} U^{\dagger}\right)\left(\partial_{\theta_{j}} U\right)$ by Eq. (3). This value can be estimated by a Hadamard test circuit, shown in Fig. 2(b), where one ancillary qubit implements a controlled- $A$ operation on the system qubits and then the auxiliary is measured in the $Z$ Pauli basis to obtain $M_{i j}=-\operatorname{Im}\langle 0|A| 0\rangle=\langle Z\rangle$. Considering the conjugate symmetry, the controlled $A$ can be simplified and only two CZ gates are left.

For the vector elements $V_{i}$, they are related to the real part of an inner product $\langle 0|B(t)| 0\rangle$, where $B(t)=$ $\left(\partial_{\theta_{i}} U^{\dagger}\right) H(t) U$. Here, the controlled- $B$ operation cannot be directly implemented in a Hadamard test circuit due to the nonunitarity of the Hamiltonian $H$ is in general nonunitary. We decompose the Hamiltonian to a sum of $K$ Pauli terms as $H(t)=\sum_{l=1}^{K} h_{l}(t) P_{l}$, where each Pauli operation $P_{l}$ is unitary and $h_{l}(t)$ is the time-dependent weighting coefficient. So, we have $\langle 0|B(t)| 0\rangle=\sum_{l=1}^{K} h_{l}(t)\left\langle 0\left|B_{l}\right| 0\right\rangle$ with $B_{l}=\left(\partial_{\theta_{i}} U^{\dagger}\right) P_{l} U$, which can be directly implemented using controlled $B_{l}$ in the Hadamard test circuits. Now, we can obtain a vector element through $V_{i}(t)=$ $\operatorname{Re}\langle 0|B(t)| 0\rangle=\sum_{l=1}^{K} h_{l}(t)\langle Z\rangle_{l}$ by measuring these $K$ Hadamard test circuits [see Fig. 1(b)]. 


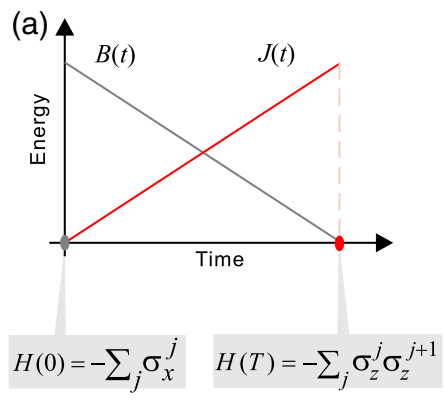

(b)

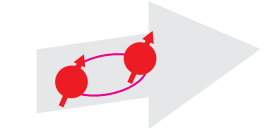

(i)

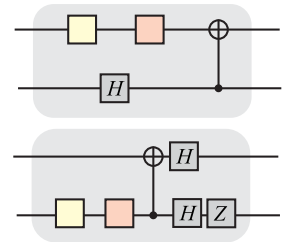

(c)

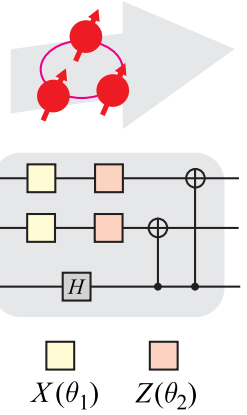

FIG. 2. The quantum Ising model simulated in the experiment. (a) The adiabatic change of system Hamiltonians. (b) The circuit ansatze for the eigenstates of the two-spin system. (i) is used for preparing the ground and the third-excited states, and (ii) is used to prepare for the first- and second-excited states. (c) The circuit ansatz for the ground-state problem of the fully coupled three-spin system.

In addition, we note another estimating method for vector element $V_{i}$, that does not use the Hadamard test. Because of Eq. (3), we have

$$
V_{i}(t)=\frac{1}{2} \frac{\partial}{\partial \theta_{i}}(\langle\phi(\vec{\theta})|H| \phi(\vec{\theta})\rangle)
$$

This vector element can be approximated by a finite difference of the Hamiltonian expectation $\langle H\rangle$, which can be measured directly on the ansatz circuit. So, we can obtain a vector element by measuring $2 K$ Pauli expectations.

Therefore, for each step, the measurement complexity on quantum computers to update the ODE's coefficients is $O\left(L^{2}\right)$ and $O(L K)$ for $M$ and $V$, respectively, for a quantum ansatz of $L \sim \operatorname{poly}(N)$ parameters and a Hamiltonian of $K \sim \operatorname{poly}(N)$ Pauli terms. Combining the computation complexity $O\left(L^{2}\right)$ of the numerical integration on classical computers, our hybrid method is efficient to solve the quantum dynamics of an $N$-qubit system.

In our experiment, we focus on the 1D Ising model under a transverse magnetic field for proof-of-principle demonstration. The time-dependent Hamiltonian with a periodic boundary condition is

$$
H(t)=B(t) H_{0}+J(t) H_{T},
$$

with $H_{0}=-\sum_{j} \sigma_{x}^{j}$ and $H_{T}=-\sum_{j} \sigma_{z}^{j} \sigma_{z}^{j+1}$. We set $J(t)=t / T$ and $B(t)=1-t / T$, which represent the strength of spin-spin interactions and the strength of the magnetic field, respectively. The change of the Hamiltonian over the whole time period is shown in Fig. 2(a).

We first consider the Ising model of two spins. In various experiments we start from the ground, first, second, and third eigenstates of the Hamiltonian $H_{0}$, given by $|++\rangle$, $(|+-\rangle \pm|-+\rangle) / \sqrt{2}$, and $|--\rangle$, respectively. The circuit ansatze are shown in Fig. 2(b), which can cover the quantum state manifold of full adiabatic paths. There are parameters $\theta_{1}$ and $\theta_{2}$ in the circuits, controlling two singlequbit gates with a rotation angle around the $X$ and $Z$ axis, respectively. The circuit ansatze are helpful to simplify our Hadamard test circuits while keep enough degrees of freedom to test the algorithm.

In our experiments, we modified the algorithm's work flow to speed up the classical-quantum iterations. We in advance estimated the ODE's coefficients in the whole ansatz parameter space on the quantum coprocessor, so the classical integral can run continually by fast querying these coefficients. The coefficients are generated in two steps. (1) We first measure the coefficients on a discrete $20 \times 20$ grid of the parameter space (from 0 to $2 \pi$ with a step size $\pi / 10)$. (2) We then linearly interpolate the results of each circuit to approximate the results that do not reside on the grid.

The experiment was conducted in a superconducting quantum processor of four working transmon qubits [20,21] (also see Fig. S1 in Supplemental Material for details [22]). The energy relaxation time $T_{1}$ (dephasing time $\left.T_{2}^{*}\right)$ are 34.6, 39.1, 29.6, and 28.1 (3.4,4.3, 5.6, and 3.1) $\mu \mathrm{s}$, respectively. The single-qubit rotation gate [23] and two-qubit fast adiabatic controlled phase (CZ) gates [24] are implemented in 30 and $60 \mathrm{~ns}$, and have average gate fidelities about 0.998(1) and 0.99(2) from randomized benchmarking [25], respectively. The qubit readout errors are $3.1 \%-9.4 \%$ for $|0\rangle$ states and $10.1 \%-16.2 \%$ for $|1\rangle$ states, which are corrected by the inverse positive operatorvalued measure (POVM) matrices to generate a corrected distribution [26].

We first applied the algorithm to the ground-state dynamics. At each grid point $\left(\theta_{1}, \theta_{2}\right)$, we measured the value of $M_{12}, V_{1}$, and $V_{2}$ by Hadamard test circuits, which are shown in Fig. S3 [22]. These circuits were compiled to run on the quantum coprocessor and the basic process of circuit compiling is illustrated in Fig. S2 [22]). We measured each circuit 5000 times to reduce the statistical noise. We plot the measured $M_{12}$ in Fig. 3(a) and $V_{1}(t)$ and $V_{2}(t)$ in Figs. 3(b) and 3(c) at time $t=T / 2$.

We then used iterative numerical integration $\vec{\theta}(t+\delta t)=$ $\vec{\theta}(t)+M^{-1} \cdot V(t) \times \delta t$ to solve the parameter trajectories on a classical computer. The time step is set as $\delta t=0.01$. 
(a)
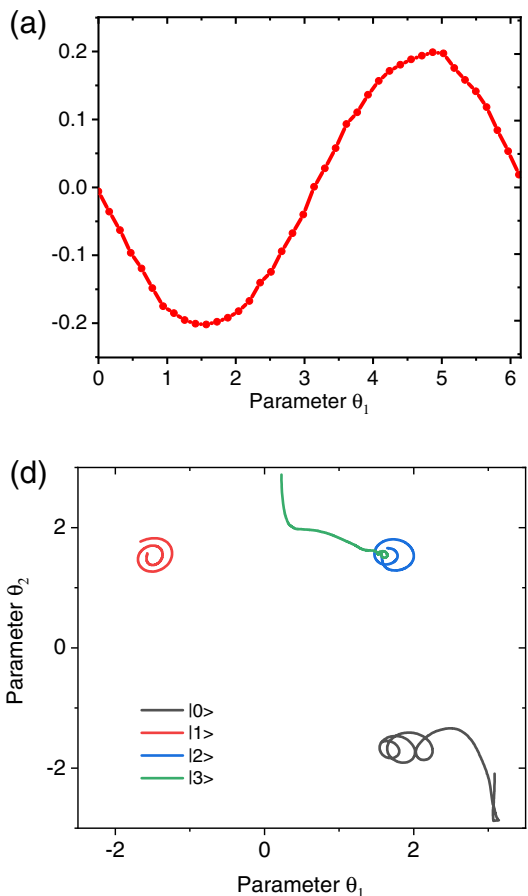

(b)
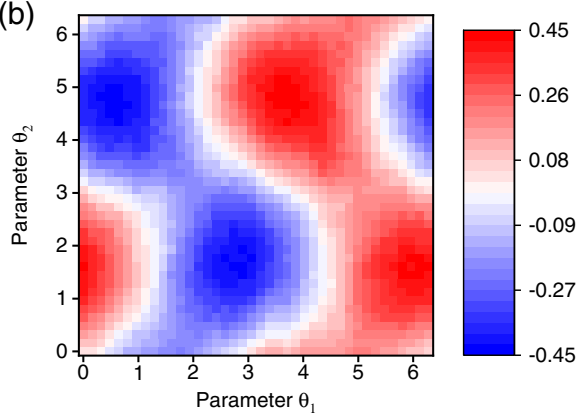

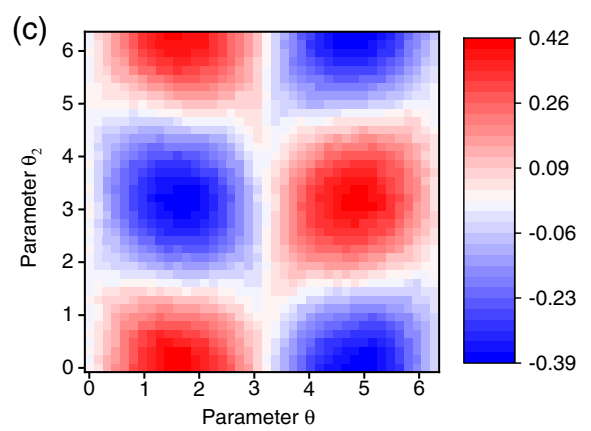

(e)
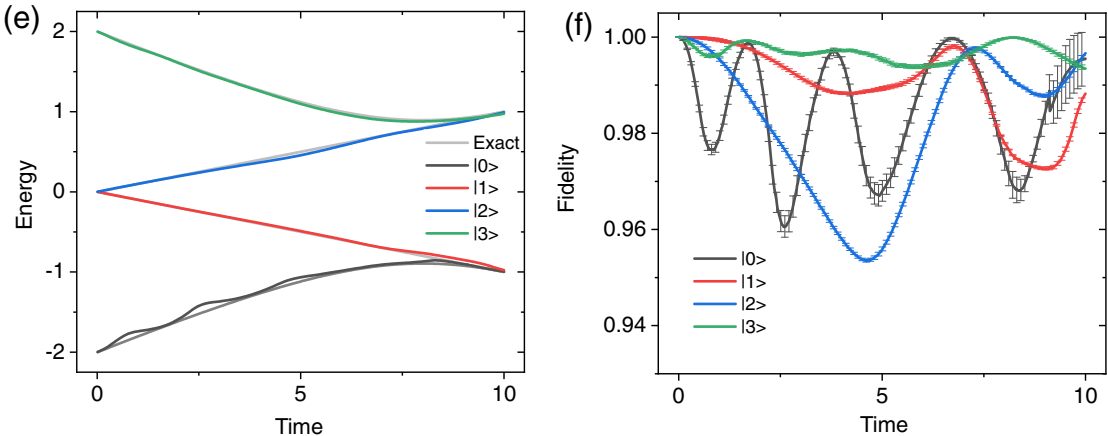

FIG. 3. Experimental results of adiabatic variational dynamics of two spins. (a) The measured $M_{12}$. Note that $M_{12}$ is only dependent on $\theta_{1}$ after the circuit compiling; $M_{11}$ and $M_{22}$ are always zero and $M_{21}=-M_{12}$ due to the symmetry from Eq. (3). (b),(c) The measured $V_{1}(t)$ and $V_{2}(t)$ at time $t=T / 2$. (d) The simulated parameter trajectories of all four eigenstates. (e),(f) The calculated energies and quantum state fidelities during the dynamics. The error bars are produced from 100 Monte Carlo simulations of the statistical fluctuation of 5000 samples of each circuit.

The calculated parameter trajectories are shown in Fig. 3(d), including the other three excited states.

We further evaluated the evolution of the system's energies and quantum state fidelities on a classical computer by using the solved parameters. It reveals the quality of the core adiabatic variational routine by excluding the quantum state preparation and measurement errors. The results are shown in Figs. 3(e) and 3(f). In Fig. 3(e), we observe that the experimental results agree well with the system eigenspectrum at all times, which verifies the effectiveness of the adiabatic property. In Fig. 3(f), we find that the average state fidelities are above $95 \%$ with systematic oscillations.

We note that the fast oscillation is a phenomenon of nonideal adiabatic following: due to the noise in the quantum circuit, the dynamical ansatz does not fully match the instantaneous eigenstate of the system Hamiltonian. This oscillating departure does not expand during the evolution. In particular, the fidelities of the four eigenstates at time $T$ are $99.5 \%, 98.8 \%, 99.6 \%$, and $99.3 \%$, respectively.

Next, we continue to investigate the evolution of the ground state of a three-spin Ising Hamiltonian. The ground states of $H_{0}$ and $H_{T}$ are $|+++\rangle$ and $(|000\rangle+|111\rangle) / \sqrt{2}$, corresponding to paramagnetic and ferromagnetic states, respectively. The circuit ansatz to simulate the adiabatic transition is shown in Fig. 2(c). Unlike the previous twoqubit ansatze, the current ansatz does not exactly cover all the quantum states during the evolution. As shown in Fig. 4(f), the ideal fidelities in the intermediate evolution are less than $97 \%$. So, it provides an interesting case to investigate the effect of adiabatic following across a restricted subspace of the ansatz.

We measured the matrix $M$ through Hadamard test circuits and measured the vector $V$ using a finite difference of Eq. (4). That is, $V_{i}(t)=\sum_{l=1}^{K} h_{l}(t)\left\langle\partial_{\theta_{i}} P_{l}\right\rangle / 2$, with

$$
\left\langle\partial_{\theta_{i}} P_{l}\right\rangle \approx \frac{\left\langle\phi\left(\vec{\theta}+\delta \cdot \vec{e}_{i}\right)\left|P_{l}\right| \phi\left(\vec{\theta}+\delta \cdot \vec{e}_{i}\right)\right\rangle-\left\langle\phi(\vec{\theta})\left|P_{l}\right| \phi(\vec{\theta})\right\rangle}{\delta},
$$

where $\delta=0.2$ is the increment of parameter $\theta_{i}, \vec{e}_{i}$ is the unit vector along $\theta_{i}$, and $P_{l}$ is the Pauli term in the Hamiltonian $H(t)=\sum_{l=1}^{K} h_{l}(t) P_{l}$. The circuits are shown in Fig. S5 [22] and the results are shown in Figs. 4(a)-4(c). In Fig. 4(d), we plot the parameter trajectory solved on the classical computer with time step $\delta t=0.01$.

We plot the calculated energy and state fidelity of the evolving state in Figs. 4(e) and 4(f), respectively. We observe the expected characteristic oscillation in the evolution due to nonideal adiabatic following. We also verify the unexpected phenomenon: although the expressive ability of the ansatz is limited in the middle time, the evolution of quantum state fidelity recovers in the end. The final state fidelity is $98.9 \%$. It is an interesting question to further investigate the nonmonotonicity of quantum state 
(a)

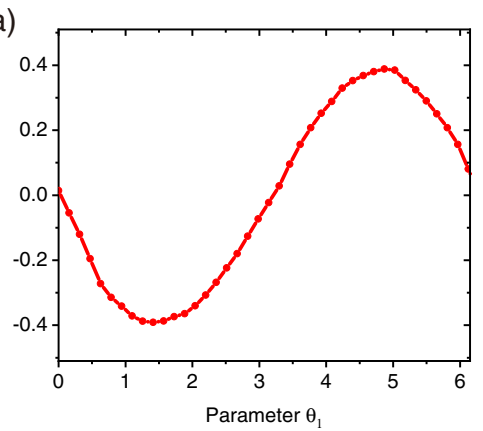

(d)

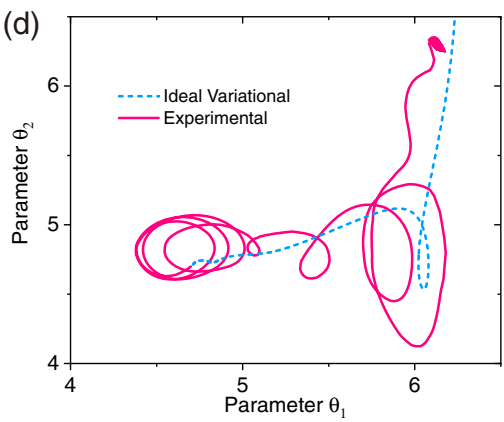

(b)
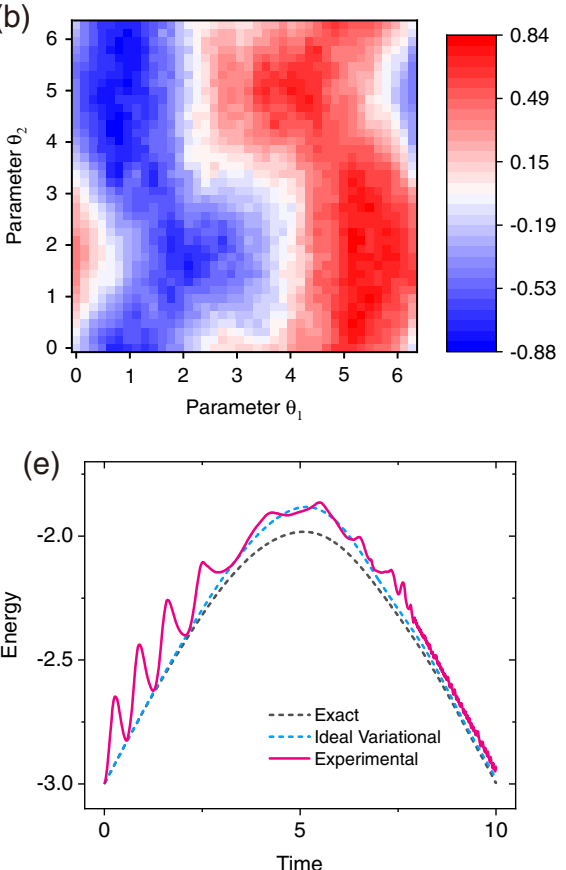

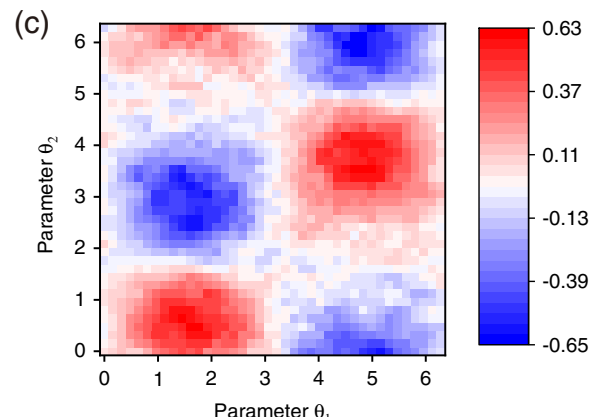

(f)

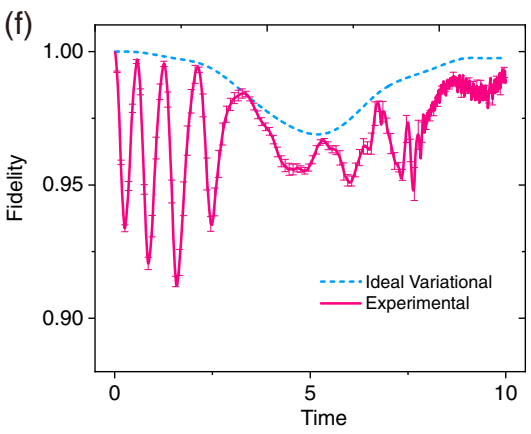

FIG. 4. Experimental results of the adiabatic variational dynamics of three spins. (a) The measured $M_{12} . M_{12}$ is only dependent on $\theta_{1}$ after the circuit compiling; $M_{11}$ and $M_{22}$ are always zero and $M_{21}=-M_{12}$ due to the symmetry from the Eq. (3). (b),(c) The measured $V_{1}(t)$ and $V_{2}(t)$ at time $t=T / 2$, respectively. (d) The simulated parameter trajectory. (e),(f) The calculated evolution of ground-state energy and fidelity. The error bars are produced from 100 Monte Carlo simulations of the statistical fluctuation of 5000 samples of each circuit. We observe an interesting phenomenon that the quantum state fidelity recovers at the end of the dynamics.

fidelity in general variational dynamics from the limited expressive ability of the ansatz.

In these two basic examples, we realized a proof-ofprinciple demonstration of adiabatic variational simulations with circuit ansatz of depth 1 . The demonstrated algorithm flow can be directly applied to more generalized circuit ansatz for complex Hamiltonians. A key problem in the conventional variational algorithm with deep circuit ansatz is the barren plateaus effect will degrade the gradient-based classical optimization [27]. Our adiabatic-based algorithm has a potential to use coherent dynamics to evade this effect. We hope to test the algorithm with deeper circuit ansatz in high-fidelity experiment in the next step.

When seeking to scale the algorithm to a large quantum system, we would find that the fidelity of simulation depends on two related issues: the choice of an appropriate circuit ansatz and how to resist experimental noise. Encouraging progress is being made in both topics.

A good ansatz circuit would be informed by knowledge of the physical system being simulated, for example, the conserved symmetries [28] and the physical intuition [27,29]. Meanwhile, circuit noise can be significantly reduced by hardware-level quantum circuit compilation and suppressed with recently proposed error mitigation methods such as techniques based on extrapolating different experimental data [18,30-36].
In summary, we have reported the experimental demonstration of a variational quantum algorithm to simulate a system's adiabatic dynamics. This method avoids the high demand of quantum hardware in analog or digital adiabatic state preparation while it is superior to classical simulated annealing by exploiting quantum coherence. Our work represents a new and promising approach to quantum dynamics simulation and quantum enhanced optimization on intermediate-scale quantum computers.

We thank Ying Li for insightful discussion. This work was supported by the National Natural Science Foundation of China, the National Fundamental Research Program, the National Key R\&D Program of China, the Chinese Academy of Sciences, Shanghai Municipal Science and Technology Major Project, and the Anhui Initiative in Quantum Information Technologies. S. C. B. and X. Y. acknowledge the EPSRC National Quantum Technology Hub (EP/M013243/1 and EP/T001062/1).

M.-C. Chen, M. Gong, and X. Xu contributed equally to this work.

[1] A. Aspuru-Guzik, A. D. Dutoi, P. J. Love, and M. Head-Gordon, Science 309, 1704 (2005). 
[2] T. Helgaker, P. Jorgensen, and J. Olsen, Molecular Electronic-Structure Theory (John Wiley \& Sons, Chichester, 2014).

[3] S. McArdle, S. Endo, A. Aspuru-Guzik, S. Benjamin, and X. Yuan, Rev. Mod. Phys. 92, 15003 (2020).

[4] Y. Cao, J. Romero, J. P. Olson, M. Degroote, P. D. Johnson, M. Kieferová, I. D. Kivlichan, T. Menke, B. Peropadre, N. P. Sawaya et al., Chem. Rev. 119, 10856 (2019).

[5] B. P. Lanyon, C. Hempel, D. Nigg, M. Müller, R. Gerritsma, F. Zähringer, P. Schindler, J. T. Barreiro, M. Rambach, G. Kirchmair et al., Science 334, 57 (2011).

[6] X.-s. Ma, B. Dakic, W. Naylor, A. Zeilinger, and P. Walther, Nat. Phys. 7, 399 (2011).

[7] R. Babbush, N. Wiebe, J. McClean, J. McClain, H. Neven, and G. Kin-Lic Chan, Phys. Rev. X 8, 011044 (2018).

[8] E. L. Ulungu and J. Teghem, J. Multi-Criteria Decis. Anal. 3, 83 (1994).

[9] E. Farhi, J. Goldstone, S. Gutmann, J. Lapan, A. Lundgren, and D. Preda, Science 292, 472 (2001).

[10] P. C. Pop, C. P. Sitar, I. Zelina, and I. Taşcu, in International Conference on Combinatorial Optimization and Applications (Springer, Xi'an, 2007), pp. 154-162.

[11] E. Y. Loh, J. E. Gubernatis, R. T. Scalettar, S. R. White, D. J. Scalapino, and R. L. Sugar, Phys. Rev. B 41, 9301 (1990).

[12] S. Lloyd, Science 273, 1073 (1996).

[13] D. S. Abrams and S. Lloyd, Phys. Rev. Lett. 79, 2586 (1997).

[14] T. Albash and D. A. Lidar, Rev. Mod. Phys. 90, 015002 (2018).

[15] M. Born and V. Fock, Z. Phys. 51, 165 (1928).

[16] E. Farhi, J. Goldstone, and S. Gutmann, arXiv:1411.4028.

[17] A. Peruzzo, J. McClean, P. Shadbolt, M.-H. Yung, X.-Q. Zhou, P. J. Love, A. Aspuru-Guzik, and J. L. O'Brien, Nat. Commun. 5, 4213 (2014).

[18] Y. Li and S.C. Benjamin, Phys. Rev. X 7, 021050 (2017).

[19] X. Yuan, S. Endo, Q. Zhao, S. Benjamin, and Y. Li, Quantum 3, 191 (2019).
[20] R. Barends, J. Kelly, A. Megrant, A. Veitia, D. Sank, E. Jeffrey, T. C. White, J. Mutus, A. G. Fowler, B. Campbell et al., Nature (London) 508, 500 (2014).

[21] M. Gong, M.-C. Chen, Y. Zheng, S. Wang, C. Zha, H. Deng, Z. Yan, H. Rong, Y. Wu, S. Li et al., Phys. Rev. Lett. 122, 110501 (2019).

[22] See Supplemental Material at http://link.aps.org/ supplemental/10.1103/PhysRevLett.125.180501 for the details of quantum coprocessor, circuit compiling, and theoretical results of the measurements.

[23] F. Motzoi, J. M. Gambetta, P. Rebentrost, and F. K. Wilhelm, Phys. Rev. Lett. 103, 110501 (2009).

[24] J. Ghosh, A. Galiautdinov, Z. Zhou, A. N. Korotkov, J. M. Martinis, and M. R. Geller, Phys. Rev. A 87, 022309 (2013).

[25] C. Ryan, M. Laforest, and R. Laflamme, New J. Phys. 11, 013034 (2009).

[26] F. B. Maciejewski, Z. Zimborás, and M. Oszmaniec, Quantum 4, 257 (2020).

[27] J. R. McClean, S. Boixo, V. N. Smelyanskiy, R. Babbush, and H. Neven, Nat. Commun. 9, 4812 (2018).

[28] J. Lee, W. J. Huggins, M. Head-Gordon, and K. B. Whaley, J. Chem. Theory Comput. 15, 311 (2018).

[29] P. K. Barkoutsos, J. F. Gonthier, I. Sokolov, N. Moll, G. Salis, A. Fuhrer, M. Ganzhorn, D. J. Egger, M. Troyer, A. Mezzacapo et al., Phys. Rev. A 98, 022322 (2018).

[30] J. R. McClean, M. E. Kimchi-Schwartz, J. Carter, and W. A. de Jong, Phys. Rev. A 95, 042308 (2017).

[31] K. Temme, S. Bravyi, and J. M. Gambetta, Phys. Rev. Lett. 119, 180509 (2017).

[32] S. Endo, S. C. Benjamin, and Y. Li, Phys. Rev. X 8, 031027 (2018).

[33] J. Colless, V. Ramasesh, D. Dahlen, M. Blok, M. Kimchi-Schwartz, J. McClean, J. Carter, W. de Jong, and I. Siddiqi, Phys. Rev. X 8, 011021 (2018).

[34] M. Otten and S. Gray, Phys. Rev. A 99, 012338 (2019).

[35] S. McArdle, X. Yuan, and S. Benjamin, Phys. Rev. Lett. 122, 180501 (2019).

[36] X. Bonet-Monroig, R. Sagastizabal, M. Singh, and T. O’Brien, Phys. Rev. A 98, 062339 (2018). 